MS15-P34 Solid state molecular packing and photoluminescence of platinum complexes based on

\section{1,5-naphthyridin-4-olate ligands}

Chin-Ti Chen ${ }^{1,2}$, Anurack Poloek ${ }^{1,3,4}$, Chieh Wang ${ }^{2}$, Chiao-Wen Lin $^{1}$, Chao-Tsen Chen ${ }^{4}$

1. Institute of Chemistry, Academia Sinica

2. Department of Applied Chemistry, National Chiao Tung University

3. Nano Science and Technology Program, TIGP, Academia Sinica

4. Department of Chemistry, National Taiwan University

email: chintchen@gate.sinica.edu.tw

Four heteroleptic platinum complexes, FPtdmaND, FPtOPhND, FPtCzND, and FPtpxzND, have different molecular packing patterns, which affect their photoluminescence (PL) in solution and in solid state or doped thin film (of PS, CBP, 4P-NPB). Interestingly, these platinum complexes exhibited varied emission from greenish yellow to orange red wavelengths due to their monomeric and aggregate/excimeric emission, which is either substitutent of 1,5-Naphthyridin-4-olate ligand (ND), concentration of dopant, or host material dependent.

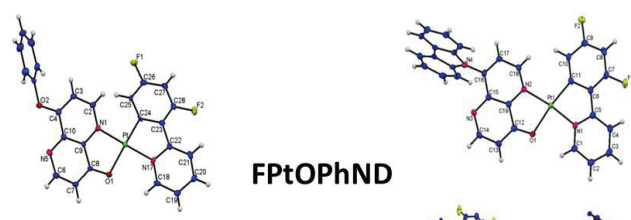

FPtCZND
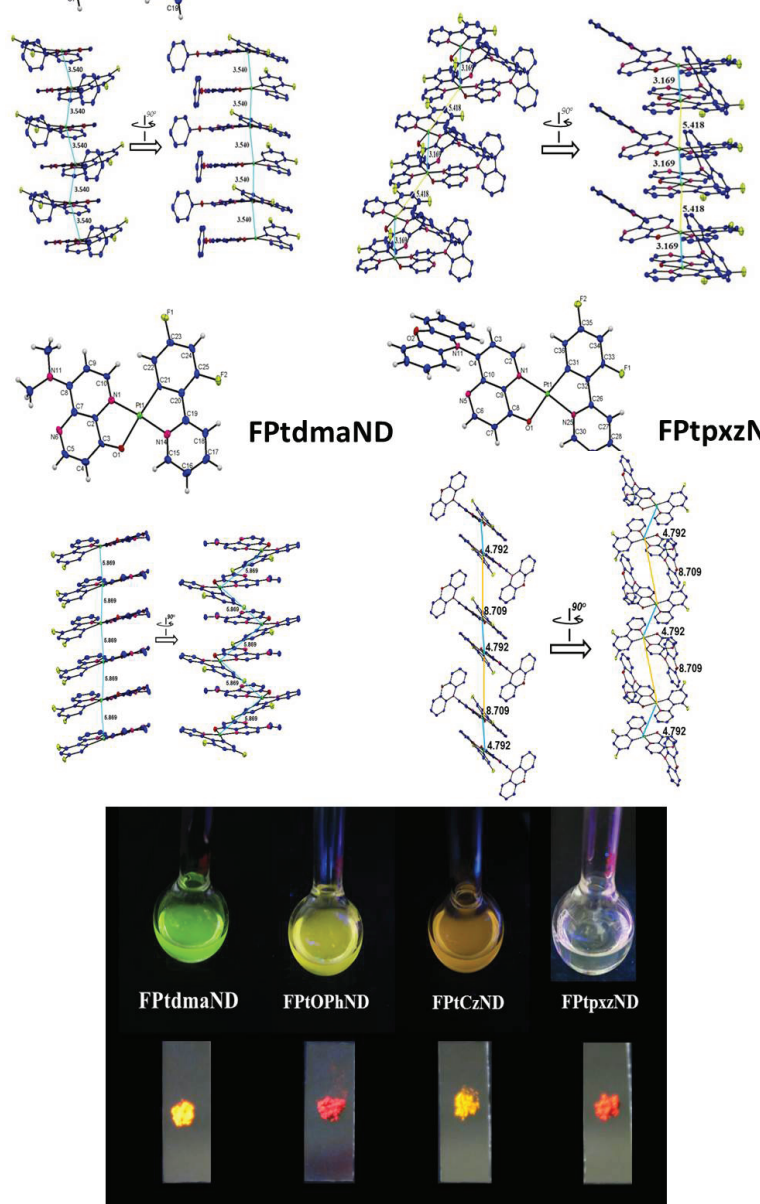

Figure 1. X-ray crystal molecular packing structure and photoluminescence image of four platinum complexes

Keywords: Molecular packing, Photoluminescence, Platinum complexes 